\title{
Orals
}

\section{Oral papers}

001

The impact of the COVID-19 pandemic on people with psoriasis: findings from a global patient-reported registry

S. Mahil, ${ }^{1}$ M. Yates, ${ }^{2}$ Z. Yiu, ${ }^{3}$ S. Langan, ${ }^{1,4}$ T. Tsakok, ${ }^{1}$ N. Dand, ${ }^{2}$ K. Mason, ${ }^{5}$ H. McAteer, ${ }^{6}$ F. Meynell, ${ }^{1}$ B. Coker, ${ }^{7}$ A. Vincent, ${ }^{7}$ D. Urmston, ${ }^{6}$ A. Vesty, ${ }^{6}$ J. Kelly, ${ }^{3}$ C. Lancelot, ${ }^{8}$ L. Moorhead, ${ }^{9}$ H. Bachelez, ${ }^{10}$ F. Capon, ${ }^{2}$ C. Romina Contreras, ${ }^{11}$ C. De La Cruz, ${ }^{12}$ P. Di Meglio, ${ }^{2}$ P. Gisondi, ${ }^{13}$ D. Jullien, ${ }^{14}$ J. Lambert, ${ }^{15}$ L. Naldi, ${ }^{16}$ S. Norton, ${ }^{17}$ L. Puig, ${ }^{18}$ P. Spuls, ${ }^{19}$ T. Torres, ${ }^{20}$ R. Warren, ${ }^{3}$ H. Waweru, ${ }^{8}$ J. Weinman, ${ }^{21}$ C. Griffiths, ${ }^{3}$ J. Barker, ${ }^{22}$ M. Brown, ${ }^{7}$ J. Galloway ${ }^{23}$ and C. Smith ${ }^{1}$

${ }^{1}$ St John's Institute of Dermatology, Guy's and St Thomas' NHS Foundation Trust and King's College London, London, UK; ${ }^{2}$ King's College London, London, UK; ${ }^{3}$ Dermatology Centre, Salford Royal NHS Foundation Trust, The University of Manchester, Manchester, UK; ${ }^{4}$ Faculty of Epidemiology, and Population Health, London School of Hygiene and Tropical Medicine, London, UK; ${ }^{5}$ School of Medicine, Keele University, Keele, UK; ${ }^{6}$ The Psoriasis Association, Northampton, UK; ${ }^{7}$ NIHR Biomedical Research Centre at Guy's and St Thomas' NHS Foundation Trust and King's College London, London, UK; ${ }^{8}$ International Federation of Psoriasis Associations, Bromma, Sweden; ${ }^{9}$ Guy's and St Thomas' NHS Foundation Trust, London, UK; ${ }^{10}$ Department of Dermatology, AP-HP Hôpital Saint-Louis, Paris, France; ${ }^{11}$ Catedra de Dermatologia, Hospital de Clinicas, Facultad de Ciencias Medicas, Universidad Nacional de Asuncion, Paraguay; ${ }^{12}$ Clinica Dermacross, Santiago, Chile; ${ }^{13}$ Section of Dermatology and Venereology, University of Verona, Verona, Italy; ${ }^{14}$ Department of Dermatology, Edouard Herriot Hospital, Hospices Civils de Lyon, University of Lyon, Lyon, France; ${ }^{15}$ Department of Dermatology, Ghent University, Ghent, Belgium; ${ }^{16}$ Centro Studi GISED, Bergamo, Italy; ${ }^{17}$ Psychology Department, Institute of Psychiatry, Psychology and Neuroscience, King's College London, London, UK; ${ }^{18}$ Department of Dermatology, Hospital de la Santa Creu i Sant Pau, Universitat Autònoma de Barcelona, Barcelona, Spain; ${ }^{19}$ Department of Dermatology, Amsterdam Public Health/Infection and Immunology, Amsterdam University Medical Centers, Location AMC, Amsterdam, The Netherlands; ${ }^{20}$ Department of Dermatology, Centro Hospitalar do Porto, Portugal, Portugal; ${ }^{21}$ School of Cancer and Pharmaceutical Sciences, King's College London, London, UK; ${ }^{22}$ St John's Institute of Dermatology, School of Basic and Medical Biosciences, Faculty of Life Sciences and Medicine, King's College London, London, UK; and ${ }^{23}$ Centre for Rheumatic Diseases, King's College London, London, UK

S.M. and M.Y. are joint first authors.

The COVID-19 pandemic has led to uncertainty over immunosuppressant-related infection risks, shortfalls in the care of long-term conditions and increased psychiatric illness. People with psoriasis may be particularly vulnerable to the negative effects of the pandemic owing to prevalent physical and psychiatric multimorbidity, and the need for systemic immunosuppressants in moderate-to-severe disease. This study sought to assess the course of psoriasis in the pandemic and characterize the factors associated with worsening disease. Individuals with clinician-confirmed psoriasis completed the online global self-report registry Psoriasis Patient Registry for Outcomes, Therapy and Epidemiology of COVID-19 Infection Me (PsoProtectMe), up to 7 September 2020. Participants specified if their psoriasis had worsened, improved or remained the same in the pandemic. Each participant completed validated self-report screens for depression (Patient Health Questionnaire-2) or anxiety (Generalised Anxiety Disorder2). Odds ratios (OR) and 95\% confidence intervals (CI) for the association between worsening psoriasis and demographic and disease-specific factors were estimated using multivariable logistic regression. In total, 3049 people with psoriasis (and without confirmed/suspected COVID-19) from 72 countries self-reported to PsoProtectMe [mean age 47.0 (SD 15.1) years; mean body mass index $27.5 \mathrm{~kg} \mathrm{~m}^{-2}$ (SD 6.0); 2003 (65.7\%) patients were female; and 2396 (78.6\%) were of white European ethnicity]. Altogether, $1219 \quad(40.0 \%)$ participants reported worsening of their psoriasis in the pandemic. There was an inverse association between worsening psoriasis and male sex (OR 0.49, 95\% CI 0.40-0.61). Worsening disease was associated with a positive screen for depression or anxiety [adjusted OR (aOR) 1.96, 95\% CI 1.55-2.47], obesity (aOR $1 \cdot 24$, 95\% CI 1.09-1.41), shielding/quarantine (aOR 1.26, 95\% CI 1.08-1.47) and systemic therapy nonadherence (aOR $3 \cdot 27,95 \%$ CI $2 \cdot 57-4 \cdot 17)$. The most common reason for nonadherence was concern about complications related to COVID$19(65 \cdot 8 \%)$. These data suggest a substantial burden from worsening psoriasis in the pandemic. To mitigate potentially long-lasting effects of the pandemic on health outcomes in psoriasis, access to holistic care should be maintained, including psychological and weight-management support. Patient nonadherence to therapy may be addressed through evidencebased communication on treatment-related COVID-19 risks.

\section{2 \\ Doxycycline in the management of bullous pemphigoid: real-world data from a specialist centre} D. Micallef $f^{1,2}$ and K.E. Harman ${ }^{2}$

${ }^{1}$ Mater Dei Hospital, Msida, Malta and ${ }^{2}$ University Hospitals of Leicester NHS Trust, Leicester, UK

The Bullous Pemhigoid Steroids and Tetracyclines (BLISTER) trial, published in 2017, showed doxycycline $200 \mathrm{mg}$ daily to be noninferior to prednisolone $0.5 \mathrm{mg} \mathrm{kg}^{-1}$ daily as the initial treatment of bullous pemphigoid (BP) in terms of efficacy at 6 weeks. In addition, there were significantly fewer adverse events in the doxycycline group at 52 weeks (Williams HC, Wojnarowska F, Kirtschig G et al. Doxycycline versus prednisolone as an initial treatment strategy for bullous pemphigoid: a pragmatic, non-inferiority, randomised controlled 